\title{
Los MOOC están muy vivos. Respuestas a algunas preguntas
}

\section{The MOOC are very alive. Some questions answered}

\author{
Lorenzo García Aretio \\ UNED (España)
}

\section{Resumen}

En un anterior trabajo (García Aretio, 2015) adelantábamos muchas dudas sobre los MOOC, un total de 90 interrogantes. En este artículo editorial sintetizamos ahora esos interrogantes en los siguientes nueve epígrafes: (a) las bases conceptuales; (b) cuestiones metodológicas; (c) la docencia, tipologías y tareas; (d) perfiles de los participantes y sus actitudes; (e) calidad, rendimiento académico y abandono; (f) universidad y MOOC; (g) soportes tecnológicos, el vídeo; (h) economía y MOOC, y (i) revisiones de la literatura científica sobre MOOC. Pues bien, en RIED han sido publicados hasta la fecha un total de 30 artículos relacionados con MOOC, de los que aquí sintetizamos numerosas respuestas valiosas a los citados interrogantes.

Palabras clave: MOOC; TIC; educación a distancia; criterios de calidad; educación superior; formación virtual; evaluación; innovación.

\begin{abstract}
In a previous work (Garcia Aretio, 2015), we expressed doubts about the MOOC phenomenon using 90 questions. In this editorial, we summarize these questions in the following nine headings: a) the conceptual basis; b) methodological issues; c) teaching, types and tasks; d) participants profiles and attitudes; e) quality, academic performance and school drop-out; f) university and MOOCs; g) technological support and video; h) economy and MOOCs; and i) reviews of the scientific literature on MOOCs. In RIED a total of 30 articles related to MOOCs have been published to date. Here we summarize many valuable answers provided therein to the questions abovementioned.
\end{abstract}

Keywords: MOOCs; ICT; distance education; quality criteria; higher education; online learning; assessment; innovation.

A finales de 2014, publicado en 2015, escribíamos un artículo editorial en RIED (García Aretio, 2015) en el que nos hacíamos eco del fenómeno MOOC, de sus repercusiones sociales, académicas y económicas que se venían debatiendo. En dicho trabajo nos posicionábamos frente a quienes consideraban que los MOOC estaban comenzando a morir y que en poco tiempo pasarían a formar parte de la historia 
de la educación como una evolución de los formatos a distancia que no llegaría a consolidarse.

Ya entonces considerábamos que esta propuesta innovadora estaba viva, muy viva, aunque los iniciales lineamientos estuviesen siendo muy matizados según los tipos de proyectos institucionales o de políticas educativas. Nos quedamos con la afirmación de Cooperman (2014) en la que afirma que en 1970, Martin Trow, profesor de la Universidad de California, Berkeley, identificó una transición "en camino en cada sociedad avanzada, de la elite a la educación superior de masas y, posteriormente, a un acceso universal". Ahí, en esa transición, evidentemente, se encuentran los MOOC.

Sin embargo, por la heterogeneidad de propuestas MOOC; por la diversidad de resultados de las investigaciones, en algunos casos no muy concordantes; por los diferentes enfoques metodológicos aplicados; por los variados matices que a los propios términos identificadores del fenómeno (curso, masivo, abierto, en línea) se le han ido agregando; por los diversos énfasis sobre los beneficios de los MOOC que en las propuestas se han venido reforzando, etc. Por todo ello, en el artículo citado más arriba nos planteábamos una amplia serie de preguntas a las que convendría dar respuesta. Algunas de ellas podrían ser de contestación sencilla, otras requerirían de amplio debate, análisis más detenidos y rigurosos.

Pues bien, con esa inquietud que mantuvimos desde la misma aparición de los MOOC, tratamos de impulsar estudios científicos que pudieran ver la luz en una revista especializada en los formatos educativos configurados en espacios virtuales. La RIED. Revista Iberoamericana de Educación a Distancia fue muy sensible al fenómeno. Una de las revistas científicas más preocupada por el tema. Lo decimos porque desde 2012 hasta este último número de RIED, el vol. 20(1), hemos publicado en la revista un total de 30 trabajos científicos sobre el tema. No podía ser de otra manera, en una revista nacida para estudiar y profundizar en todas las innovaciones y evoluciones de propuestas educativas relacionadas con los formatos no presenciales y con las metodologías sustentadas en diferentes tecnologías. Los MOOC se han convertido en un fenómeno que algunos consideran como disruptor y que para nosotros suponen una evolución, eso sí, muy drástica, de la educación a distancia que, considerada desde un enfoque amplio y abarcador, para nosotros, sí que fue, sí que es, disruptora. Ya en 2014, la RIED era una de las revistas españolas con mayor número de publicaciones sobre el fenómeno MOOC, considerado ampliamente, como prueba la tabla 1 que mostramos (Aguaded, Vázquez-Cano y López-Meneses, 2016). 
Tabla 1. Revistas y editoriales con mayor índice de publicación en MOOC

\begin{tabular}{|c|c|}
\hline Revista / Editor & $\%$ \\
\hline Revista de Currículum y Formación del Profesorado & 26,19 \\
\hline RIED. Revista Iberoamericana de Educación a Distancia & $\mathbf{1 1 , 9 0}$ \\
\hline Digital Education Review & 9,52 \\
\hline RED. Revista de Educación a Distancia & 7,14 \\
\hline Comunicar & 4,76 \\
\hline Revista de Educación y Derecho = Education and Law Review & 4,76 \\
\hline RUSC. Revista de Universidad y Sociedad del Conocimiento & 4,76 \\
\hline Anuario ThinkEPI & 2,38 \\
\hline @tic: Revista d’Innovació Educativa & 2,38 \\
\hline Ediciones Universidad de Salamanca & 2,38 \\
\hline GECONTEC: Revista Internacional de Gestión del Conocimiento y la Tecnología & 2,38 \\
\hline Historia y Comunicación Social & 2,38 \\
\hline IJERI: International Journal of Educational Research and Innovation & 2,38 \\
\hline Innovación Educativa & 2,38 \\
\hline Journal for Educators, Teachers and Trainers & 2,38 \\
\hline Octaedro & 2,38 \\
\hline REDU: Revista de Docencia Universitaria & 2,38 \\
\hline Síntesis & 2,38 \\
\hline Teoría de la Educación & 2,38 \\
\hline Universidad Internacional de La Rioja (UNIR) & 2,38 \\
\hline
\end{tabular}

Fuente: Aguaded, Vázquez-Cano y López-Meneses (2016)

Como después comprobaremos, hasta 2014 se habían publicado en RIED un total de 7 artículos (1 en 2013 y el resto en 2014), considerando que el de 2013 fue recibido en RIED en 2012 (año de los MOOC). Sin embargo, hasta el momento actual, incluyendo el número de RIED al que pertenece este trabajo, han sido publicados en esta revista, desde 2013, un total de 30 trabajos científicos, incluido el que ahora lee. La Revista de Currículum y Formación del Profesorado que aparece a la cabeza de esa tabla 1, publicó en 2014 un monográfico, el 18(1), sobre MOOC que recogió en el mismo número un total de 24 trabajos. Sin embargo, posteriormente, y hasta la fecha de redactar este trabajo, buscando en la propia revista con el término "MOOC", solo hemos encontrado un artículo más, correspondiente al número 19(3), de 2015.

La RIED, más que otras revistas, ha sentido la responsabilidad de atender desde una perspectiva científica el fenómeno MOOC, porque es una revista internacional cuyo eje es la educación a distancia en cualesquiera de sus diferentes formulaciones y dimensiones. Y a los MOOC los venimos encuadrando ahí desde el principio, como última propuesta en la progresiva evolución de los formatos educativos que se alejan 
de las propuestas presenciales. Esa preocupación de RIED se ha plasmado en la publicación de tres monográficos, en 2014, otro en 2015 y el actual, correspondiente a enero de 2017. Además de ello, se han publicado otros artículos relacionados con el fenómeno en otros números no monográficos. En total, 30 trabajos relacionados de una u otra forma con los MOOC, desde el año 2013 hasta enero de 2017. La tabla 2 muestra la relación de artículos de RIED ordenados por año.

Vamos a tratar de agrupar estos 30 trabajos en función de las pretensiones de dar respuesta a algunas de aquellas preguntas que nos hacíamos a finales de 2014.

Tabla 2. Artículos publicados en RIED con temas relacionados con los MOOC

\begin{tabular}{|c|c|c|c|}
\hline & TÍTULO & AÑO & VOL. \\
\hline A & 1. Proposições e controvérsias no conectivismo & 2013 & 16,2 \\
\hline G & $\begin{array}{l}\text { 2. El mini video como recurso didáctico en el aprendizaje de } \\
\text { materias cuantitativas }\end{array}$ & 2013 & 16,2 \\
\hline $\mathrm{C}$ & $\begin{array}{l}\text { 3. Figura de los facilitadores en los Cursos Online Masivos y } \\
\text { Abiertos (COMA/MOOC): nuevo rol profesional para los entornos } \\
\text { educativos abiertos }\end{array}$ & 2014 & 17,1 \\
\hline B & $\begin{array}{l}\text { 4. Los MOOC y su papel en la creación de comunidades de } \\
\text { aprendizaje y participación }\end{array}$ & 2014 & 17,1 \\
\hline B & 5. Entornos abiertos en la nueva educación a distancia & 2014 & 17,1 \\
\hline $\mathrm{F}$ & $\begin{array}{l}\text { 6. From elite to mass to universal higher education: from distance to } \\
\text { open education. }\end{array}$ & 2014 & 17,1 \\
\hline $\mathrm{F}$ & $\begin{array}{l}\text { 7. Analysis of successful modes for the implementation and use of } \\
\text { OCW \& OER in higher education. The virtual mobility case }\end{array}$ & 2014 & 17,1 \\
\hline $\mathrm{F}$ & $\begin{array}{l}\text { 8. Los Cursos Online Masivos y Abiertos: ¿oportunidad o amenaza } \\
\text { para las universidades iberoamericanas? }\end{array}$ & 2014 & 17,1 \\
\hline B & $\begin{array}{l}\text { 9. Adaptatividade geocultural em ambientes virtuais de } \\
\text { aprendizagem }\end{array}$ & 2014 & 17,1 \\
\hline A & 10. MOOC: ¿tsunami, revolución o moda pasajera? & 2015 & 18,1 \\
\hline $\mathrm{D}$ & 11. Percepción de los participantes sobre el aprendizaje en un MOOC & 2015 & 18,2 \\
\hline $\mathrm{E}$ & 12. Criterios de calidad para la valoración y gestión de MOOC & 2015 & 18,2 \\
\hline $\mathrm{E}$ & 13. La valoración de MOOC: una perspectiva de calidad & 2015 & 18,2 \\
\hline A & 14. La filosofía educativa de los MOOC y la educación universitaria & 2015 & 18,2 \\
\hline B & $\begin{array}{l}\text { 15. MOOC: ecosistemas digitales para la construcción de PLE en la } \\
\text { educación superior }\end{array}$ & 2015 & 18,2 \\
\hline A & 16. Accesibilidad y MOOC: hacia una perspectiva integral & 2015 & 18,2 \\
\hline A & 17. Visiones educativas sobre los MOOC & 2015 & 18,2 \\
\hline $\mathrm{E}$ & $\begin{array}{l}\text { 18. Validación del cuestionario de evaluación de la calidad de cursos } \\
\text { virtuales adaptado a MOOC }\end{array}$ & 2015 & 18,2 \\
\hline
\end{tabular}




\begin{tabular}{|l|l|r|r|}
\hline & \multicolumn{1}{|c|}{ TÍTULO } & AÑO & VOL. \\
\hline B & $\begin{array}{l}\text { 19. A New Competence-based Approach for Personalizing MOOCs in } \\
\text { a Mobile Collaborative and Networked Environment }\end{array}$ & 2016 & 19,1 \\
\hline A & $\begin{array}{l}\text { 20. } \text { Elearning, educação online e educação aberta: contributos para } \\
\text { uma reflexão teórica }\end{array}$ & 2016 & 19,1 \\
\hline G & $\begin{array}{l}\text { 21. Metodología de producción para el desarrollo de contenidos } \\
\text { audiovisuales y multimedia para MOOC }\end{array}$ & 2017 & 20,1 \\
\hline I & $\begin{array}{l}\text { 22. Analysis of the scientific literature on Massive Open Online } \\
\text { Courses (MOOCs)/ }\end{array}$ & 2017 & 20,1 \\
\hline I & $\begin{array}{l}\text { 23. The academic production on open educational resources in } \\
\text { Portuguese }\end{array}$ & 2017 & 20,1 \\
\hline H & 24. Cursos MOOC: un enfoque desde la economía & 2017 & 20,1 \\
\hline D & $\begin{array}{l}\text { 25. La atención a las demandas específicas del alumnado en un } \\
\text { mundo globalizado el caso de un MOOC de español para viajar }\end{array}$ & 2017 & 20,1 \\
\hline I & $\begin{array}{l}\text { 26. La productividad científica sobre MOOC: aproximación } \\
\text { bibliométrica 2012-2016 a través de SCOPUS }\end{array}$ & 2017 & 20,1 \\
\hline F & $\begin{array}{l}\text { 27. ¿Pueden los MOOC favorecer el aprendizaje y hacer disminuir las } \\
\text { tasas de abandono universitario? }\end{array}$ & 2017 & 20,1 \\
\hline E & $\begin{array}{l}\text { 28. MOOC: medición de satisfacción, fidelización, éxito y certificación } \\
\text { de la educación digital }\end{array}$ & 2017 & 20,1 \\
\hline I & 29. Visión de los MOOCs desde una perspectiva práctica & 2017 & 20,1 \\
\hline A & 30. Los MOOC muy vivos. Respuestas a algunas preguntas & 2017 & 20,1 \\
\hline
\end{tabular}

Ya decíamos antes que hace dos años (Tabla 2, $\mathrm{n}^{0}$ 10) adelantábamos nuestras dudas, que redactábamos en formato de 51 preguntas, que si quedaban desglosadas se convertían en un total de unos 90 interrogantes. En ese mismo artículo tratábamos de agrupar esas inquietudes sobre los MOOC en estos nueve epígrafes o bloques que ahora adaptamos al momento actual y que remarcamos en la primera columna de la tabla 2:
A. Las bases conceptuales.
B. Cuestiones metodológicas.
C. La docencia. Tipologías y tareas.
D. Perfiles de los participantes y actitudes.
E. Calidad. rendimiento, abandonos, indicadores de calidad y eficacia.
F. Universidad y $M O O C$.
G. Soportes tecnológicos. El vídeo.
H. Economía y MOOC.
I. Revisiones de la literatura científica sobre MOOC. 
Trataremos de encontrar respuestas a estos diferentes bloques en los que distribuimos aquellas preguntas. Iremos desbrozando bloque a bloque y ofreciendo los principales resultados, reflexiones o conclusiones de los diferentes artículos publicados en esta revista RIED de los que entresacamos textos literales de los mismos. Resulta evidente que podríamos abarcar un mayor espectro de revistas o de bases de datos para redondear este trabajo. Pero solo nos vamos a limitar a revisar los artículos de RIED que resultan una muestra interesante de lo publicado a nivel internacional. Vayamos a ello.

\section{A. Las bases conceptuales}

Con el artículo de Somoza (Tabla 2, $\mathrm{n}^{0}$ 1) se abre este capítulo de trabajos publicados en RIED relacionados con este tema de estudio. La autora discute sobre las ideas y aplicaciones del proyecto conectivista aduciendo las controversias existentes entre los defensores y los críticos de esa corriente. Los MOOC se visualizaron en sus inicios muy ligados al conectivismo. Sin embargo, Soares llega a dudar incluso de sus aportes al mundo de los c-MOOC o MOOC conectivistas solicitando esfuerzos en la investigación de estos modelos. Aprovecha para mostrar las características del primer MOOC conectivista (Downes, 2008) para realizar un análisis del mismo.

En nuestro trabajo, ya referido más arriba, decididamente frente a otros criterios, apostábamos porque los MOOC suponían una evolución de la educación a distancia y no una ruptura. Son muchas las características de esta modalidad que también perfilan los rasgos esenciales de un MOOC. Tratamos, en ese nuestro trabajo, de responder brevemente a lo que fue el nacimiento y primeros desarrollos de los MOOC. Por otra parte, complementábamos el artículo con otras innovaciones relacionadas que propiciaron el nacimiento de estos cursos masivos. Nos referimos a los Recursos Educativos Abiertos (REA - Open Educational Resources - OER) que supusieron embriones de la apertura del conocimiento ofrecido, entre otros espacios, desde prestigiosas instituciones de ámbito mundial como el Instituto Tecnológico de Massachusetts (MIT) a través de su iniciativa Open Course Ware (OCW).

Respecto a la asunción de los MOOC en los ambientes educativos, (Tabla, $2 \mathrm{n}^{\mathrm{o}}$ 17) Cabero habla de hacerlo de forma reflexiva y crítica, integrándolos como una tecnología más, que los docentes tenemos a nuestra disposición para crear una verdadera escenografía virtual para la formación en la sociedad del conocimiento. Se trataría de otra tecnología más, que la persona incorpore a su entorno personal de aprendizaje y que al docente le permita crear un verdadero ecosistema para la formación virtual. Insiste el autor en la necesidad de dar respuesta, a través de la investigación, a cuestiones vitales, buena parte de ellas coincidentes con las explicitadas por García Aretio en su citado trabajo. Concluye Cabero que los MOOC han llegado para quedarse, pero que la visión futura que tengamos de ellos va a ser diferente a las visiones actuales que tenemos de los mismos. Los MOOC no son recetas mágicas que van a resolver los problemas educativos de la sociedad 
del conocimiento; no son para todo el mundo ni para todos los estudiantes; no van a resolver los problemas de las instituciones educativas, pero sí facilitan un nivel de acceso sin precedentes y son una interesante oportunidad para crear nuevas escenografías educativas.

Cabero asume la definición sobre MOOC de Marauri (2014, p. 40)

... un curso gratuito, en abierto, compuesto fundamentalmente por Recursos Educativos Abiertos (REA) y diseñado para poder ser cursado, a través de una plataforma o entorno personal de aprendizaje instalado en la red Internet, por cualquier persona, de manera autónoma, sin necesidad de contar con un profesor o tutor de apoyo en red al otro lado de la conexión.

Y describe las características distintivas de estos cursos así como las diferencias entre los dos tipos más clásicos generalmente admitidos, los xMOOC y cMOOC, determinadas en que los primeros persiguen que los alumnos adquieran una serie de contenidos y tienden a ser las mismas versiones de los cursos en e-learning pero ubicados en las plataformas específicas de los MOOC y los segundos se apoyan en una idea conectivista de la enseñanza, y de acuerdo con ello, el conocimiento no se centra en los expertos, sino en las conexiones que establecen los estudiantes que participan en la acción formativa. Por su parte, Del Moral y Villalustre (Tabla 2, $\mathrm{n}^{0} 15$ ) entienden que un MOOC de calidad es un ecosistema digital versátil y personalizable, capaz de responder a las demandas de los usuarios, permitiéndoles organizar su propio aprendizaje de forma divergente y creativa atendiendo a sus preferencias cognitivas, y dispensándoles el asesoramiento experto durante todo el proceso, que contempla un eficaz sistema de comunicación para facilitar el intercambio de información y la elaboración colaborativa del conocimiento.

En aspectos teóricos también incursionan Torres y Gago (Tabla 2, $\mathrm{n}^{0} 4$ ), al referirse a las tipologías de MOOC, reiteradas en la literatura científica al respecto. Estos autores señalan que el valor más prometedor de los MOOC no deriva de lo que ahora son sino de lo que el tiempo nos confirmará que llegarán a ser, precisamente por sus capacidades de modularidad, escalabilidad y capacidad de recombinación y por las derivadas positivas que están empezando a aflorar y que tienen mucho que ver con el carácter flexible y abierto del aprendizaje que preconizan. En consecuencia, una reconfirmación de la vitalidad de los MOOC.

En esta línea, Teixeira y otros (Tabla 2, $\mathrm{n}^{0}$ 19) afirman que los MOOC combinan la propiedad de ser abiertos con la posibilidad de ser escalables de una forma muy poderosa. Tienen el potencial de permitir la participación en la educación superior a todas las personas y a todos los niveles. Por lo tanto, contribuyen a la inclusión social, la difusión del conocimiento y la innovación pedagógica, así como a la internalización de las instituciones de educación superior.

Por su parte, Aires (Tabla 2, $\mathrm{n}^{0}$ 20) en un trabajo que trata de abordar la multiplicidad de enfoques referidos a los conceptos de e-Learning, Educación online 
y Educación abierta, vinculados los tres a la idea abarcadora de Educación a distancia, se refiere a los Recursos Educativos Abiertos (REA) y a los MOOC. En relación a los REA destaca que la matriz de ese movimiento sobrepasa el mero acceso a contenidos y recursos y se asocia a una nueva filosofía educativa, a nuevos valores basados en la apertura, en la ética de la participación y en la colaboración. En el ámbito de los MOOC, Aires destaca una ecología de aprendizaje específica de esos cursos MOOC, y hace hincapié en la necesidad de reconceptualizar propuestas tradicionales referidas a la inscripción, participación, programa y rendimiento de los estudiantes.

Vázquez-Cano y López-Meneses (Tabla 2, $\mathrm{n}^{0}$ 14) enfatizan que los MOOC están cuestionando principios e ideas tradicionales de la Educación como el "cocimiento enlatado", el acceso limitado, la autoridad impuesta y el paradigma científicoracionalista en pro de una ecología más dinámica del conocimiento. Tanto para alumnos como profesores, se abren nuevas puertas al conocimiento, aunque "todo lo que reluce no es oro" y el movimiento necesita afrontar algunas controversias y retos como la autentificación, acreditación y los altos índices de abandono.

Finalmente, el concepto de masividad y apertura, parecería que debería englobar la "accesibilidad". En este caso, Rodríguez-Ascaso y González Boticario (Tabla 2, n ${ }^{\circ}$ 16) proponen un marco de servicios, estándares, normas de calidad y consideraciones que deberían atenderse si se pretenden cubrir necesidades de cada persona, también de quienes tienen alguna discapacidad. Se realiza una recopilación de requisitos de usuarios con y sin discapacidad en instituciones de educación superior que utilizan tecnología. A continuación, y basándose en las metodologías de diseño centrado en el usuario, se propone un conjunto de escenarios para ilustrar las necesidades de cualquier usuario MOOC, y las limitaciones derivadas de la falta de apoyo que actualmente se presta a la diversidad funcional de esos estudiantes MOOC. En el trabajo se apuntan las principales líneas de actuación presentes y futuras, en las que se está trabajando para la detección de estados afectivos del estudiante que condicionan su proceso de aprendizaje y que podrían utilizarse, por ejemplo, para intentar reducir los ratios significativos de abandono que se experimentan en los MOOC.

\section{B. Cuestiones metodológicas}

Respecto a los aspectos referidos a las diferentes variables curriculares, encontramos a Torres y Gago (Tabla 2, $\mathrm{n}^{\circ} 4$ ), que ligan los MOOC a sus posibilidades de generar comunidades de aprendizaje y participación, a través de su integración dentro de entornos "paraguas", constituidos por comunidades en donde los participantes puedan diseñar sus propios entornos de aprendizaje de acuerdo con sus competencias y sus necesidades. Esas comunidades tendrán como uno de sus protagonistas a los MOOC, en su concepción más originaria de uso exclusivo del canal online. Estos entornos "paraguas", como Colmenia, dan cobertura a algunas de las tendencias novedosas por las que los MOOC ya están transitando en la actualidad, 
pero, sobre todo, lo harán en el inmediato futuro. El concepto de comunidad de comunidades descrito en este trabajo de Torres y Gago constituye un extraordinario escenario para enriquecer el enfoque transmedia incorporando al mundo de la educación nuevas realidades de formación.

Adaptar los cursos masivos a las diferentes realidades geoculturales referida a la procedencia diversa de los estudiantes es otro problema recurrente al que Palazzo y otros (Tabla 2, $n^{\circ}$ 9) aproximan alguna respuesta, al tratar de contribuir al desarrollo masivo dela inclusión digitaly delas habilidades de aprendizaje delas personas a través de la construcción de un sistema digital sensible a las características geoculturales de los participantes en el curso. Los autores analizan esas características con el fin de aumentar la eficacia de los ambientes virtuales de aprendizaje para grandes grupos de estudiantes. Esa preocupación por las características de alumnos procedentes de diferentes regiones es uno de los mayores empeños para el diseño e implementación de MOOC con ambición de internacionalidad. Los cursos que se ofrecen tienen una enorme diversidad cultural y, por lo tanto, deben estar preparados para adaptar sus estrategias de enseñanza y estructuras de navegación y contenidos a las diferentes culturas de los usuarios.

Una respuesta a lo anterior nos la parecen ofrecer Teixeira y otros (Tabla $2, \mathrm{n}^{\mathrm{o}}$ 19) al afirmar que uno de los elementos críticos para que tenga éxito una experiencia de aprendizaje MOOC es la de potenciar y facilitar una red de aprendizaje. De hecho, los MOOC no están diseñados para un número predefinido de participantes por lo que sirven para un alto número de perfiles heterogéneos, con diversidad de estilos de aprendizaje y conocimientos previos, pero también contextos de participación y diversidad de plataformas online. La personalización puede desempeñar un papel clave en este proceso.

El modelo pedagógico iMOOC del que hablan Teixeira y otros introdujo el principio de diversidad en el diseño de MOOC, permitiendo una clara diferenciación de caminos de aprendizaje y también entornos virtuales. En este artículo los autores presentan una propuesta basada en este enfoque de iMOOC, como un nuevo sistema para la personalización y adaptación de MOOC diseñados en un enfoque colaborativo y en una red pedagógica. Los iMOOC implican una adaptación completa, no solo para el perfil personal del participante, sino también para los dispositivos móviles utilizados. Importan en el modelo iMOOC la innovación, la interacción, la inclusión, la responsabilidad individual y las relaciones interpersonales.

Del Moral y Villalustre (Tabla 2, $\mathrm{n}^{0} 15$ ) afirman que los MOOC posibilitan a los estudiantes universitarios el acceso a la información y al conocimiento de forma personalizada, constituyéndose en experiencias formativas únicas para la construcción y gestión de sus propios Entornos Personales de Aprendizaje (PLE), propiciando la utilización de recursos y herramientas adaptables a sus estilos cognitivos. En este sentido, los autores proponen una serie de indicadores destinados a determinar la calidad y adaptabilidad de los MOOC como nuevos ecosistemas digitales que ofrecen diversas posibilidades para el aprendizaje y la comunicación. 
Se trataría de definir qué es un MOOC de calidad y determinar los aspectos que deben incorporar sus diseños para favorecer la creación de esos Entornos Personales de Aprendizaje que incrementen su satisfacción, garantizando su permanencia y compromiso con su culminación. Para ello, los autores elaboraron un instrumento que les sirvió para identificar las condiciones y rasgos que contribuyen a convertir los MOOC en espacios favorecedores para la construcción de PLE, integrado por 30 ítems agrupados en torno a 5 dimensiones de análisis: (a) acceso y organización de contenidos, (b) mecanismos para propiciar el aprendizaje, (c) comunicación social y formativa, (d) creación y publicación de elaboraciones personales y (e) colaboración en tareas de producción colectiva.

Por otra parte, este nuevo tipo de aprendizaje plantea nuevos retos a las universidades y profesores: redefinir el actual contexto metodológico hacia un diseño más interactivo, colaborativo y de materiales ubicuos, e implementar nuevas formas de autoevaluación más dinámicas.

\section{La docencia. Tipología y tareas}

De forma más específica, Marauri (Tabla 2, $\mathrm{n}^{\mathrm{o}} 3$ ) ha sido el autor, de entre los analizados, que más espacio ha dedicado a una de las figuras docentes más visibles en los MOOC, la que él denomina como facilitadores. Marauri señala que la proliferación de los MOOC está provocando, sobre todo por el efecto masificador que su matrícula conlleva, la aparición, o mejor dicho, la modificación de un rol originalmente ocupado por los tutores ordinarios en los cursos a distancia y en línea. $\mathrm{Al}$ ser prácticamente imposible realizar una atención y seguimiento personalizado en aulas virtuales masificadas, se hace necesario modificar su perfil inicial, de tal forma que su rol sea el de seguimiento y dinamización de los mensajes existentes en el foro de debate, facilitando la resolución de las dudas que se generan y abandonando definitivamente su rol como evaluador. Las pruebas de autoevaluación y pruebas objetivas de evaluación en línea son calificadas automáticamente por el propio sistema, y las entregas individuales de actividades y tareas son evaluadas entre pares. Por tanto, observamos que la gran diferencia entre ambos roles es la ausencia total de su papel como evaluador. Es decir, un facilitador no se encarga nunca de evaluar actividades. Este seguimiento de los mensajes en el foro no significa que tenga que leer todos los mensajes que se generan en su interior, ya que en algunos cursos sería imposible, incluso para facilitadores profesionales contratados a tiempo completo, pero sí que debe estar informado de las tendencias y derivas que toma el curso, interviniendo puntualmente para reconducir los temas que se presentan y facilitando la estancia y el tránsito de los alumnos por cada uno de los módulos que lo componen. Por tanto, además de seguir la inercia del curso a través de los foros, debe contestar cualquier duda allí expuesta y solventar los problemas que se vayan presentando. 


\section{Perfiles de los participantes y actitudes}

Los resultados de una investigación centrada en la percepción de los estudiantes sobre el aprendizaje en un curso MOOC cooperativo, nos la ofrecen Castaño, Maíz y Garay (Tabla 2, $\mathrm{n}^{\mathrm{o}}$ 11). Su trabajo se centra en un curso MOOC universitario, Grado de Maestro en Educación Primaria, aunque abierto a la participación de todas las personas interesadas. La percepción de los estudiantes se evalúa a través del cuestionario TAM (Technology Acceptance Model), adaptado al contexto de aprendizaje de un MOOC. Se analizaron tres variables asociadas a la percepción de los estudiantes sobre el aprendizaje en el curso: el nivel académico de los participantes (estudiantes de grado versus no estudiantes de grado), el tipo de participación en el curso (oculto, moderadamente oculto, activo, individualista y colaborador) y la variable género. Aunque la percepción de los estudiantes en la utilidad, motivación y facilidad de uso de un curso MOOC es alta, se encuentran algunas diferencias entre las variables analizadas. Se observa que los estudiantes no de grado perciben más positivamente la metodología. Sin embargo, el tipo de participación no influye de manera significativa en la percepción de los alumnos sobre el aprendizaje. Además, los hombres puntúan significativamente más alto tanto en la motivación hacia el aprendizaje a través de un MOOC, como en la percepción de su utilidad para el aprendizaje. Los resultados obtenidos muestran una buena aceptación por parte de los estudiantes de la utilización de cursos online masivos y abiertos en contextos universitarios reglados, y sugieren una mayor aceptación en entornos de aprendizaje ligados a la formación continua y al desarrollo profesional.

Sedano (Tabla 2, $\mathrm{n}^{\circ}$ 25) presenta un estudio muy peculiar centrado en "la atención a las necesidades y demandas específicas del alumnado en un mundo globalizado: el caso de un MOOC de español para viajar", aunque la metodología seguida podría ser válida para otras experiencias e implementaciones. Se trataría de analizar la atención a las expectativas y necesidades específicas de los participantes que, en este caso, aprenden una lengua con el fin específico de viajar. Los principales resultados indican que un análisis de necesidades previo para el diseño de un MOOC de estas características, con el uso de redes sociales y de REA (Recursos Educativos Abiertos) ayuda en gran medida a cumplir las expectativas de los participantes y a desarrollarse como aprendientes autónomos que podrán llevar a cabo sus acciones de lengua en un contexto globalizado. Los resultados muestran que la creación de un curso masivo de lenguas a partir de un análisis de necesidades previo, junto con la elección de esta tipología de sMOOC (social y ubicuo), con el uso de redes sociales, REA y actividades de revisión por pares $\left(\mathrm{P}_{2} \mathrm{P}\right)$, ayuda a cumplir las expectativas de los participantes que poseen una finalidad específica de aprendizaje. 


\section{E. Calidad, rendimiento, abandonos, indicadores de calidad y eficacia}

A raíz del suceso MOOC, explosivo y descontrolado, surge la duda en torno a si estos cursos y plataformas educativas poseen el sustento pedagógico adecuado que garantice la calidad y eficacia del uso de estas herramientas en la generación de aprendizaje, Aguaded y Medina-Salguero (Tabla 2, $\mathrm{n}^{\circ}$ 12). Estos autores, en su trabajo destacan que no existe una normativa específica que permita garantizar la calidad del aprendizaje en los MOOC, a pesar de que existen normativas de e-learning y que se está trabajando actualmente por aunar criterios para la evaluación de este tipo de formación online. Por otra parte, insisten estos autores, existe una gran variedad de criterios y/o dimensiones, propuestas por teóricos, a tener en cuenta en la evaluación de la calidad de los MOOC. Desde diversos organismos, instituciones y personas implicadas, se demanda la necesidad de crear estándares de calidad para evaluar a los MOOC, pero la pregunta esencial es: ¿es viable tal y como se conceptualiza este tipo de formación? Hasta el momento, según Aguaded y Medina-Salguero, los resultados nos llevan a la conclusión de que no lo es.

Sin embargo, una respuesta a los planteamientos anteriores, viene dada por la propuesta de Ramírez-Fernández (Tabla 2, no ${ }^{0}$ ) que, tras la evaluación de varios cursos a través de la herramienta de valoración EduTool $®$, concluye que queda evidenciado que los MOOC tienen una base pedagógica sólida en sus formatos. Así pues, de forma general, según este autor, se puede afirmar que la valoración de la calidad de los cursos analizados no solo está por encima de la puntuación media exigida con esta herramienta de calidad, sino que éstos presentan una puntuación promedio ligeramente superior a la media. Sin embargo, se constata que los MOOC todavía no han ensayado una ruptura con los modelos formativos online propios del e-learning. Para ello, se hacen necesarios nuevos caminos de investigación que abran de forma interdisciplinar núcleos de atención y reflexión sobre las deficiencias de los mismos. De esta manera, se podrían acreditar a las plataformas ofertantes con cursos MOOC certificados y evitar la oferta de acciones formativas con debilidades en las metodologías de enseñanza, inapropiadas desde las actuales teorías pedagógicas e impidiendo, en la medida de lo posible, la tendencia a la estandarización del conocimiento y los graves problemas para atender las diferencias individuales debido a la masificación, que conduce a un diseño comunicativo unidireccional, centrado en el docente y basado en el contenido. En todo caso, y al igual que en el trabajo que precede, el autor señala que se hace preciso continuar investigando para dar respuesta a preguntas sobre métodos que mejoren la fiabilidad, validez, autenticidad y seguridad de las evaluaciones de los usuarios, o sobre técnicas que ofrezcan evaluación automatizada eficaz y sistemas de retroalimentación inmediata.

Insisten Mengual, Roig y Lloret (Tabla 2, $\mathrm{n}^{\mathrm{O}} 18$ ) en la necesidad de instrumentos que avalen la calidad de los MOOC desde diferentes perspectivas. En este trabajo se abunda en la dimensión pedagógica, ya que de ello dependerá el éxito y la 
consolidación de este tipo de e-learning. Por ello, los autores pusieron el empeño en validar un cuestionario para la evaluación pedagógica de los MOOC. Se concluye en el trabajo que han de considerarse minuciosamente las diferentes especificidades de los MOOC, ya que éstos no pueden analizarse única y exclusivamente desde la óptica general del e-learning anterior a los MOOC. Se enfatizan así estas dimensiones de evaluación: 1) la calidad de la comunicación y los elementos multimedia de los cursos masivos en línea; 2) la coherencia curricular de los cursos y el grado de adaptación al usuario; y 3) la calidad de su planificación didáctica. Los resultados evidencian la fiabilidad de lo que los autores denominan "Cuestionario de evaluación de la calidad de cursos virtuales adaptado a MOOC". Los resultados coinciden con algunos de los principios que apunta Bates (2014) sobre cuáles deben regir el diseño de los MOOC, entre los que se destacan: comunicación, diversidad - por cuanto adaptación- y materiales de apoyo.

González de la Fuente y Carabante (Tabla 2, $\mathrm{n}^{\circ}$ 28) presentan un trabajo de alta validez y fiabilidad con más de 3000 cuestionarios respondidos. Muestran resultados altamente positivos de estudiantes de la plataforma MiriadaX, referidos a la satisfacción de expectativas (más del 90\%), la recomendación del curso (más del 80\%) y el interés en continuar cursando MOOC (más del 90\%). Quizás resulta destacable que el recurso mejor valorado es el vídeo que acompañan a los cursos, así como las pruebas de autoevaluación, considerando que deberían cuidarse más los foros. Cerca del $60 \%$, por su parte, estaría interesado en obtener un certificado oficial de pago.

\section{F. Universidad y MOOC}

Cooperman (Tabla 2, $\mathrm{n}^{0}$ 6) adapta el marco del desarrollo histórico y estructural de la educación superior como un proceso gradual en el que el crecimiento absoluto y relativo de la matrícula universitaria transforma las instituciones de educación superior y altera sus funciones. La transición hacia el acceso universal puede apoyar el desarrollo económico, la movilidad social y una mayor igualdad de ingresos, a su vez apuntalar incluso la institución de la democracia. Llegar a esos resultados sociales óptimos, afirma Cooperman, no es automático. La entrada continuada de nuevas capas sociales a la educación superior, presenta nuevos desafíos que el "acceso" por sí solo podría no resolver.

La educación de masas y el acceso universal al mismo tiempo ha venido impulsándose a través de la educación abierta y a distancia, como palancas gemelas para la reducción de costes y promoción de la calidad de la educación. Precisamente, afirma Cooperman que los políticos, si son inteligentes, solo pueden reconocer que la economía de escala promovida por los MOOC, se convierte en una herramienta ideal para erradicar la pobreza, los problemas educativos y sociales reales, los ambientales globales, los de salud pública, etc., tratando de hacer que "la educación para todos" se convierta en una realidad. 
La educación universitaria ligada a los recursos educativos abiertos REA es abordada por Tovar y Lesko (Tabla 2, $\mathrm{n}^{0}$ 7) que trataron de identificar prácticas de su implantación y uso, así como, en particular, cómo se podía facilitar la movilidad virtual de estudiantes. El trabajo forma parte del proyecto "Open Course Ware $(O C W)$ in the European HE context", y su objetivo se basa en la creación de precondiciones para un entorno fuerte europeo OCW y como consecuencia, romper los obstáculos entre instituciones europeas y por ello incrementar la movilidad real de estudiantes. Hay una serie de factores los cuales deben ser considerados con respecto a la implementación exitosa y uso de OCW en la Educación Superior. El apoyo institucional y las actitudes positivas del profesorado han sido identificados como los facilitadores más importantes para este tipo de iniciativas, pero también pueden actuar como inhibidores importantes en cuanto a la falta de apoyo institucional. Como resultados de este mismo trabajo, se afirmaba que los OCW/ OER podrían facilitar la movilidad virtual de los estudiantes.

Sobre el abandono de los estudios universitarios se pronuncia Aguado (Tabla $2, \mathrm{n}^{0}$ 27) en el sentido de que esta circunstancia puede producirse por situaciones y motivaciones muy variadas, afectando en mayor medida a los estudios que se imparten en la modalidad a distancia, y suponiendo un coste tanto para los alumnos como para la sociedad. Con el objetivo de mejorar el aprendizaje y reducir la tasa de abandono de los alumnos, se utilizó un MOOC como instrumento de apoyo a la docencia universitaria, con lo que se les facilitaban materiales escritos, vídeos explicativos, tests en cada módulo, un foro para comunicarse con compañeros y profesor y enlaces a materiales complementarios. Los resultados obtenidos fueron muy positivos; comparando las calificaciones de los alumnos entre el curso 2012/13 y el curso 2013/14, se apreció que el porcentaje de alumnos que no han superado la asignatura (suspensos o no presentados) se redujo a la mitad, de un 53\% a un $27 \%$. Así, señala Aguado, que la utilización de un MOOC como complemento de la formación reglada se ha revelado como una oportunidad muy positiva.

El idioma en el que los MOOC están siendo elaborados es determinante a la hora de alcanzar la masa crítica que este nuevo modelo de educación a distancia necesita para su sostenibilidad. Capdevilla y Aranzadi (Tabla 2, $\mathrm{n}^{\circ}$ 8) centran su atención en las universidades iberoamericanas que, según ellos, tienen frente a sí una gran oportunidad para satisfacer la demanda de conocimiento de los cientos de millones de hispano y luso parlantes del mundo que no hablen inglés y por tanto no puedan seguir los cursos de las universidades anglosajonas. En respuesta a este nuevo reto se puso en marcha a principios del 2013 Miríada X, la plataforma de Cursos Online Masivos y Abiertos con vocación iberoamericana.

\section{G. Soportes tecnológicos. El vídeo}

Gértrudix, Rajas y Álvarez (Tabla 2, $\mathrm{n}^{0}$ 21), a través de la aplicación de un proceso inspirado en los principios de investigación-acción, presentan un modelo 
metodológico para la producción y desarrollo de contenidos audiovisuales y multimedia para MOOC. El proceso se llevó a cabo durante el desarrollo secuencial de los cinco primeros MOOC elaborados para la plataforma URJCx de la Universidad Rey Juan Carlos. El proceso ha requerido de procesos iterativos y participados de diseño, implantación (acción), análisis y evaluación dirigidos a la mejora y la eficacia de los procesos de producción. La secuencia de interacciones ha permitido definir un modelo de producción más ligero, basado en el desarrollo de esquemas y patrones formales que funcionan como plantillas generales. Esta metodología, según estos autores, acaba caracterizándose por dos aspectos centrales: una alta dependencia del análisis y el seguimiento de los procesos y resultados de su aplicación; y una hibridación de enfoques y técnicas de producción que persiguen el ajuste a calendarios y presupuestos, evitar la repetición de errores y hacer evolucionar el modelo de desarrollo asociado al diseño de un producto educativo también en constante evolución para adaptarse a la demanda y al perfil de alumnado cambiantes.

Ya en 2013, de la Fuente, Montserrat y Para (Tabla 2, $\mathrm{n}^{0} 2$ ) adelantaban la fuerza del mini-vídeo en las propuestas a distancia. Estos autores muestran la aplicación de vídeos de corta duración en la enseñanza a distancia de la matemática financiera y extraen una serie de conclusiones que se refieren a los principios básicos que consideran debe reunir el vídeo para conseguir unos resultados óptimos de aprendizaje por parte de los estudiantes. También dan a conocer la opinión de los estudiantes sobre el beneficio que les ha proporcionado este tipo de herramienta con unos resultados que ponen de manifiesto la utilidad de la misma en estos formatos educativos.

\section{H. Economía y MOOC}

Concebidos los MOOC, inicialmente, como bienes libres accesibles a cualquiera en cualquier sitio, han promovido un debate en torno a su éxito y potencial beneficio económico, Rabanal, (Tabla 2, $\mathrm{n}^{0}$ 24). El trabajo de esta autora, aplicando los principios básicos de la teoría económica, hace una reflexión sobre el mercado de MOOC y los elementos determinantes de su demanda y oferta desde una perspectiva económica. El mercado de cursos MOOC se perfila como un mercado de bienes económicos públicos cercano a la competencia perfecta en sus características en el que la demanda y oferta toman la forma de los bienes económicos públicos en base a criterios cualitativos como la calidad y el altruismo. El beneficio directo o indirecto de esos cursos se fundamenta en una nueva forma de negocio donde las fuentes de ingresos están diversificadas y son analizadas. Al igual que en cualquier mercado, la oferta y demanda y su libre juego determinan su éxito, encontrándonos con que la calidad del curso es un factor esencial que juega un papel semejante al del precio de los bienes en un mercado. Muchas universidades, especialmente las españolas, han visto en estos cursos una fórmula exclusivamente económica, basando su estrategia 
en la copia en "versión española" de la estructura metodológica y didáctica de los cursos anglosajones, dejando de lado la calidad.

\section{Revisiones de la literatura científica sobre MOOC}

Los estudios sobre Recursos Educativos en Abierto (REA) proporcionan nuevas perspectivas sobre la circulación y producción de contenidos educativos impresos y digitales. Los REA son componentes importantes en la búsqueda de modelos educativos emergentes y sostenibles, como, por ejemplo, los libros didácticos abiertos y los MOOC, Zancanaro y Amiel, (Tabla 2, no 23). Este estudio tiene como objetivo describir y analizar la literatura académica en el idioma portugués sobre REA publicada hasta mayo de 2015, usando como fuente de consulta diversas bases de datos y revistas científicas que utilizan técnicas bibliométricas. Pretenden los autores contribuir al aumento de la visibilidad de la producción académica en lenguas menos prominentes como el portugués, y fomentar la integración y la colaboración entre autores que frecuentemente abordan la misma temática ignorando los estudios sobre REA desarrollados -o en desarrollo- por otros y desde otros países y lenguas. Como otras, la lengua minoritaria portuguesa tiene una visibilidad limitada en el ámbito académico. Analizar, promover y conectar en el mundo académico la producción en una lengua minoritaria es una manera de relacionar estas obras, dentro de un debate más amplio sobre un campo emergente como REA.

De manera más concreta, Zancanaro y Domingues (Tabla 2, $\mathrm{n}^{0} 22$ ) realizan una exhaustiva revisión de las investigaciones acerca de MOOC publicadas hasta diciembre de 2014 en dos bases de datos científicas: Scopus y Web of Science. Tras el análisis de la literatura científica sobre MOOC, fue posible comprobar el estado actual de la investigación en esta área. Con este fin, este trabajo permitió (a) identificar el aumento de los estudios científicos publicados en el período de tiempo estudiado; (b) mostrar las principales fuentes en las que se publicaron las obras seleccionadas; (c) presentar los principales autores y sus instituciones; (d) señalar las palabras clave más usadas; (e) clasificar los artículos en macro-temas y (f) revelar los principales marcos teóricos utilizados en los artículos identificados. El trabajo de Zancanaro y Domíngues ofrece un marco que puede ayudar eficazmente a las personas interesadas en la ampliación de estudios y desarrollo de MOOC, además de enriquecer el debate sobre la evolución de investigaciones y tendencias en MOOC.

Siguiendo con el capítulo de revisiones, el trabajo más actual de Mengual, Vázquez y López (Tabla 2, $\mathrm{n}^{\circ}$ 26) analiza la productividad científica del fenómeno MOOC a partir del análisis de 752 publicaciones indexadas en la base de datos SCOPUS en el periodo 2012-2016. Los datos extraídos permiten realizar una radiografía global del estado del fenómeno, la evolución que ha sufrido en los últimos años, el tipo de publicaciones que sustentan el fenómeno, los principales países y fuentes productores de información sobre MOOC, las principales filiaciones implicadas, los autores y trabajos más citados, así como un análisis de co-citación que permite 
vislumbrar los trabajos más destacados sobre el fenómeno. Por lo tanto, estamos ante un buen marco complementario a diversos estudios existentes de carácter nacional e internacional que intentan ofrecer y explicar la configuración del mapa de la ciencia alrededor del fenómeno MOOC. Se ha evidenciado una productividad científica destacada, que con una evaluación constante que prevé que el 2016 se posicione, si cabe, al mismo nivel que los años 2014 y 2015. En dicho sentido, este estudio coincide en resaltar que el fenómeno MOOC se consolida como área de investigación. Esta cuestión pone de manifiesto la vigencia de la temática MOOC y la confrontación con los investigadores que describen la ya era post-MOOC.

\section{Final}

Está claro que a pesar de que la bibliografía existente acerca de los $M O O C$ es muy abundante, todavía quedan sin resolver muchas incógnitas referentes a su utilidad actual y su evolución futura. Es necesario continuar realizando estudios que nos arrojen luz al respecto, especialmente si con ellos somos capaces de cuantificar y de interpretar correctamente los datos recopilados, Aguado (Tabla 2, $\mathrm{n}^{0} 29$ ). Los tres trabajos finales que recogemos en este artículo editorial nos confirman que los MOOC siguen muy vivos y que estos estudios bibliométricos son de especial interés para la comunidad académica; no solo porque fomentan la promoción de la investigación sobre los MOOC sino también porque ofrecen un panorama del crecimiento de la ciencia, en general y de un ámbito concreto, en particular.

Vamos a finalizar este trabajo corroborando la vitalidad del interés internacional sobre los MOOC presentando los Gráficos 1 y 2, extraídos de Google Trends en los que se recoge el interés de búsquedas del término referido (MOOC), desde el pasado noviembre de 2011 hasta noviembre de 2016. En el eje de abscisas se reflejan los años y en el de las ordenadas el interés de búsqueda en relación con el mayor valor de un gráfico referido a todo el mundo. Debemos aclarar que un valor de 100 indica la popularidad máxima de un término. Para visualizar mejor, hemos comparado dos términos relacionados, el e-learning con una más amplia trayectoria en su existencia $\mathrm{y}$ los MOOC. 
Gráficos 1 y 2. Google Trends. Interés de búsquedas de los términos e-learning y MOOC (nov. 2011- nov. 2016)

(dos perspectivas de la misma búsqueda)
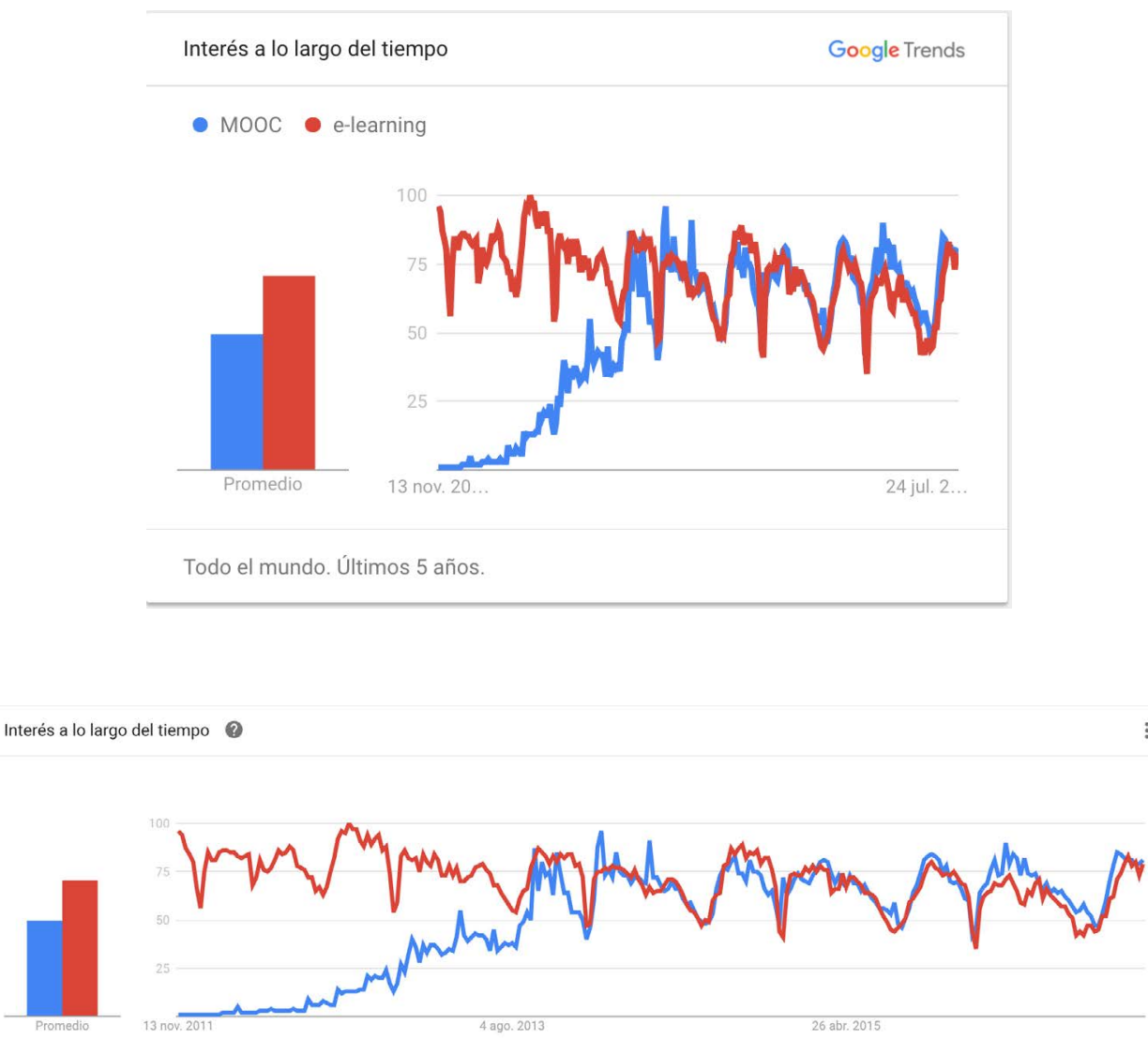

Como puede apreciarse, los gráficos presentan la popularidad de esos dos términos en los últimos cinco años, desde noviembre de 2011 hasta noviembre de 2016. El 13 de noviembre de 2011 el interés por estos términos en Google Trends estaba así: e-learning, 96 y MOOC, 1; el 21 de septiembre de 2013, el e-elarning mostraba un valor de 82 y el término MOOC, 87 . Y ya desde esa fecha, como puede apreciarse, el interés y la popularidad de estos dos términos ha ido bastante pareja. En la medida más reciente mostrada en los gráficos, 12 de noviembre de 2016, el e-learning marcaba un 79 de popularidad por un 81 de los MOOC. Hagamos la salvedad, en todo caso, de que si en lugar de comparar con "e-learning" lo hacemos con "elearning", "e-Learning”, etc. , los resultados podrían ser otros. En todo caso, 
desde septiembre de 2013, salvo algunos picos en torno a 50, buena parte del interés por las búsquedas de MOOC ha oscilado entre 75 y 100 a lo largo de estos últimos cinco años. Por tanto, llegamos a reafirmarnos en que los MOOC están muy vivos dado el interés y popularidad mostrados por esta herramienta.

\section{REFERENCIAS BIBLIOGRÁFICAS}

Aguaded, I., Vázquez-Cano, E., y LópezMeneses, E. (2016). El impacto bibliométrico del movimiento MOOC en la Comunidad Científica Española. Educación XX1, 19(2), 77-104, doi: 10.5944/educXX1.13217

Bates, A. W., y Sangrá, A. (2012). La gestión de la tecnología en la educación superior. Estrategias para transformar la enseñanza y el aprendizaje. Barcelona: Octaedro.

Downes, S. (2008). Places to go: connectvism \& connective knowledge. Innovate. Journal of Online Education, 4(6), 1-6. Nova Southeastern University. Recuperado de http:// nsuworks.nova.edu/cgi/viewcontent. cgi?article $=1037 \&$ context $=$ innovate

Nota. El resto de referencias de este trabajo, corresponden a artículos publicados en RIED (Tabla 2). Pueden consultarse en los monográficos correspondientes de los números: 16,2; 17,1; 18,2 y 19,1.

\section{Como citar este artículo:}

García Aretio, L. (2017). Los MOOC están muy vivos. Respuestas a algunas preguntas. RIED. Revista Iberoamericana de Educación a Distancia, 2O(1), (version preprint). doi: http://dx.doi.org/10.5944/ried.20.1.17488 